\title{
Astaxanthin overproduction in yeast by strain engineering and new gene target uncovering
}

Jin Jin ${ }^{1,2+}$, Ying Wang ${ }^{1,2+}$, Mingdong Yao ${ }^{1,2}$, Xiaoli Gu ${ }^{1,2}$, Bo Li $i^{1,2}$, Hong Liu ${ }^{1,2}$, Mingzhu Ding ${ }^{1,2}$, Wenhai Xiao ${ }^{1,2^{*}}$ (D) and Yingjin Yuan ${ }^{1,2}$

\begin{abstract}
Background: Astaxanthin is a natural carotenoid pigment with tremendous antioxidant activity and great commercial value. Microbial production of astaxanthin via metabolic engineering has become a promising alternative. Although great efforts have been conducted by tuning the heterologous modules and precursor pools, the astaxanthin yields in these non-carotenogenic microorganisms were still unsatisfactory for commercialization, indicating that in addition to targeted tailoring limited targets guided by rationally metabolic design, combining more globe disturbances in astaxanthin biosynthesis system and uncovering new molecular mechanisms seem to be much more crucial for further development. Since combined metabolic engineering with mutagenesis by screening is a powerful tool to achieve more global variations and even uncover more molecular targets, this study would apply a comprehensive approach integrating heterologous module engineering and mutagenesis by atmospheric and room temperature plasma (ARTP) to promote astaxanthin production in Saccharomyces cerevisiae.
\end{abstract}

Results: Here, compared to the strain with $\beta$-carotene hydroxylase (CrtZ) from Alcaligenes sp. strain PC-1, involving new CrtZ from Agrobacterium aurantiacum enhanced astaxanthin yield to 1.78-fold and increased astaxanthin ratio to $88.7 \%$ (from 66.6\%). Astaxanthin yield was further increased by 0.83 -fold (to $10.1 \mathrm{mg} / \mathrm{g}$ DCW) via ARTP mutagenesis, which is the highest reported yield at shake-flask level in yeast so far. Three underlying molecular targets (CSS1, YBR012W-B and DAN4) associated with astaxanthin biosynthesis were first uncovered by comparative genomics analysis. To be noted, individual deletion of CSS1 can recover $75.6 \%$ improvement on astaxanthin yield achieved by ARTP mutagenesis, indicating CSS1 was a very promising molecular target for further development. Eventually, $217.9 \mathrm{mg} / \mathrm{L}$ astaxanthin (astaxanthin ratio was $89.4 \%$ and astaxanthin yield was up to $13.8 \mathrm{mg} / \mathrm{g} \mathrm{DCW}$ ) was obtained in 5-L fermenter without any addition of inducers.

Conclusions: Through integrating rational engineering of pathway modules and random mutagenesis of hosts efficiently, our report stepwise promoted astaxanthin yield to achieve the highest reported one in yeast so far. This work not only breaks the upper ceiling of astaxanthin production in yeast, but also fulfills the underlying molecular targets pools with regard to isoprenoid microbial overproductions.

Keywords: Metabolic engineering, Astaxanthin, Saccharomyces cerevisiae, ARTP mutagenesis, Novel gene targets

\footnotetext{
${ }^{*}$ Correspondence: wenhai.xiao@tju.edu.cn

${ }^{\dagger}$ Jin Jin and Ying Wang contributed equally to this work

1 Key Laboratory of Systems Bioengineering (Ministry of Education),

School of Chemical \& Engineering, Tianjin University, No. 92, Weijin Road,

Nankai District, Tianjin 300072, People's Republic of China

Full list of author information is available at the end of the article
} 


\section{Background}

Astaxanthin, a member of carotenoid pigments with much higher antioxidant activity than other carotenoids and vitamin $\mathrm{E}$, has tremendous commercial value in the aquaculture, food, cosmetic and pharmaceutical industries $[1,2]$. In addition to currently commercial astaxanthin source by chemical synthesis or extraction from natural producers (such as the green algae or the red yeast) [3], microbial production of astaxanthin via metabolic engineering has emerged as a promising alternative $[4,5]$. In recent years, heterologous productions of astaxanthin have been successfully achieved in Escherichia coli [6-10], Saccharomyces cerevisiae [11, 12] and Corynebacterium glutamicum [13] through introduction of astaxanthin biosynthesis pathway into these microorganisms. Although great efforts have been conducted by metabolically engineering the heterologous modules and precursor pools, the highest astaxanthin yield in E. coli or S. cerevisiae was $15.1 \mathrm{mg} / \mathrm{g}$ DCW [14] and $8.10 \mathrm{mg} / \mathrm{g}$ DCW [12] so far, respectively (Table 1 ). The astaxanthin yields in these non-carotenogenic microorganisms were still unsatisfactory for commercialization, indicating that many more biological functions, such as biosynthesis, transport, storage and even tolerance, would likely to be regulated at the same time for further higher astaxanthin yields. In other words, in addition to targeted tailoring limited points guided by rationally metabolic design, combining global disturbances in astaxanthin biosynthesis system and further uncovering new molecular mechanisms seem to be much more crucial for further development.
Apart from metabolic engineering strategy based on rational design, mutagenesis followed by screening is a common strategy to improve phenotypic profiles especially for high yields and growth robustness through randomly and more globally affecting microenvironment in hosts. New molecular targets identified in mutagenesis are just valuable feedback to further rational design. Among physical and chemical methods for mutagenesis [15], an atmospheric and room temperature plasma (ARTP) method has been applied for mutagenesis of various species to obtain targeted biological features $[16,17]$. The ARTP mutation system can induce diverse breakage in plasmid DNA and oligonucleotides with variation of plasma dosage [18]. A mutant Blakeslea trispora was isolated from samples treated with ARTP, in which the lycopene accumulation was enhanced by $55 \%$ than that in the parent strain [19]. Zhao et al. [20] also reported that improved production profiles for lipids and carotenoids were obtained in oleaginous yeast Rhodosporidium toruloides by ARTP. Such perfect performances of ARTP in microorganism breeding and products enhancing enable it very likely to be beneficial for better astaxanthin accumulation.

In the meantime, it has been reported that the limited step of astaxanthin production is the pathway from $\beta$-carotene to astaxanthin (Fig. 1), in which two enzymes, $\beta$-carotene ketolase $\mathrm{CrtW}$ and $\beta$-carotene hydroxylase $\mathrm{CrtZ}$ are required [6]. It has been revealed that many bacterial CrtWs and CrtZs could utilize $\beta$-carotene as well as its hydroxylated or ketolated products as the substrate, leading to diverse carotenoid intermediate profiles which can greatly affect astaxanthin yield and ratio

Table 1 Astaxanthin production by different microorganisms

\begin{tabular}{|c|c|c|c|c|}
\hline Hosts & Fermentation level & $\begin{array}{l}\text { Astaxanthin yield (mg/g } \\
\text { DCW) }\end{array}$ & Astaxanthin ratio (\%) & References \\
\hline \multicolumn{5}{|c|}{ Non-carotenogenic microorganisms } \\
\hline \multirow[t]{6}{*}{ E. coli } & Shake-flask & 1.4 & 95 & {$[7]$} \\
\hline & Shake-flask & 1.99 & 90 & {$[6]$} \\
\hline & Shake-flask & 5.8 & N.D. & {$[8]$} \\
\hline & Shake-flask & 7.4 & 96.6 & [9] \\
\hline & Shake-flask & 8.64 & N.D. & [10] \\
\hline & Shake-flask & 15.1 & N.D. & [14] \\
\hline \multirow[t]{3}{*}{ S. cerevisiae } & Shake-flask & 4.7 & N.D. & [11] \\
\hline & Shake-flask & 8.1 & N.D. & [12] \\
\hline & $\begin{array}{l}\text { Shake-flask } \\
\text { 5-L bioreactor }\end{array}$ & $\begin{array}{l}10.1 \\
13.8\end{array}$ & 89.4 & This study \\
\hline Kluyveromyces marxianus & 5-L bioreactor & 9.97 & N.D. & {$[5]$} \\
\hline \multicolumn{5}{|c|}{ Carotenogenic microorganisms } \\
\hline Haematococcus pluvialis & Shake-flask & 77.2 & N.D. & [3] \\
\hline
\end{tabular}

N.D. not determined 


\section{Heterologous modules Engineering Mutagenesis by screening}

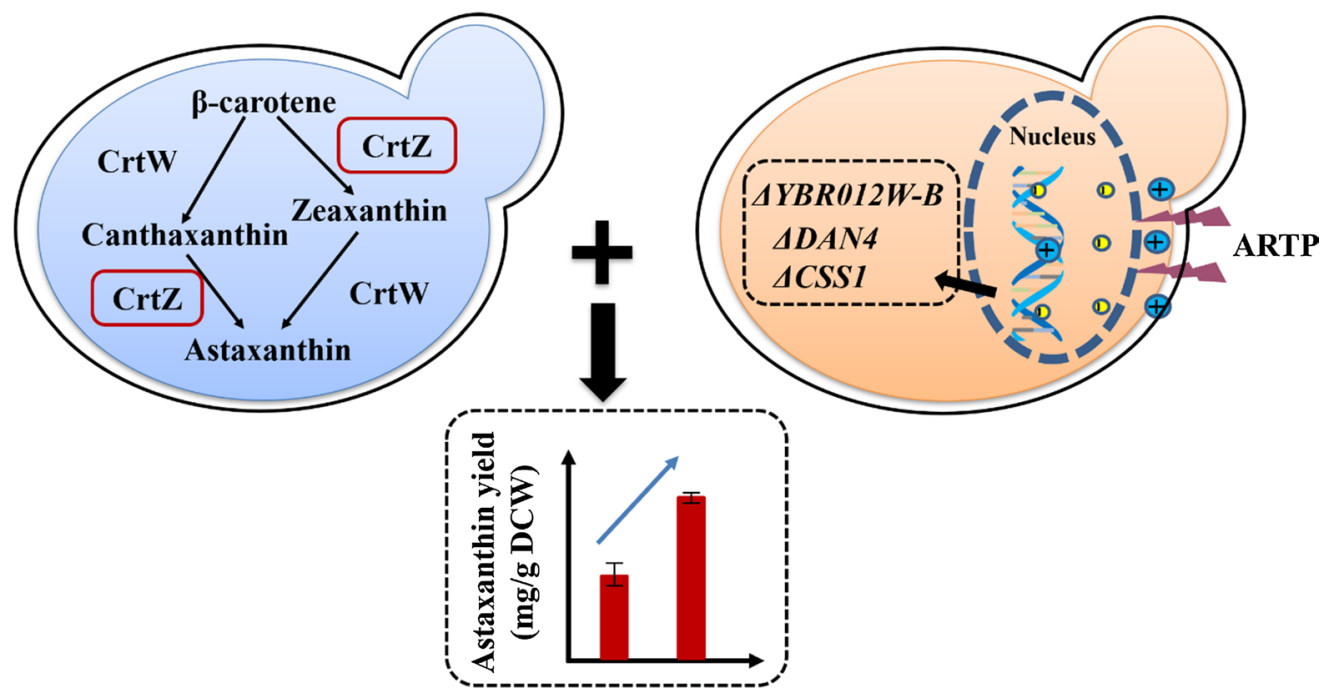

Fig. 1 Overview of astaxanthin biosynthesis pathway from $\beta$-carotene and the strategy (heterologous modules engineering combined with mutagenesis by screening) applied in this study. The targeted protein in module engineering was boxed by red. And the gene targets, that were identified to be associated with astaxanthin biosynthesis in this research, were also presented in black round square in dashed line. ARTP, atmospheric and room temperature plasma

[21, 22]. In our study, novel combination of CrtZ-CrtW with mutagenesis by ARTP process was employed to further enhance astaxanthin production and ratio in $S$. cerevisiae (Fig. 1). Consequently, an astaxanthin yield of $10.1 \mathrm{mg} / \mathrm{g} \mathrm{DCW}$ (titer of $55.7 \mathrm{mg} / \mathrm{L}$ ) at shake-flask level was obtained, which is the highest reported yield at shake-flask level in S. cerevisiae. Three promising gene targets (CSS1, YBRO12W-B and DAN4) were firstly identified from ARTP mutagenesis and their influences on astaxanthin synthesis were also validated accordingly. Finally, $217.9 \mathrm{mg} / \mathrm{L}$ astaxanthin (astaxanthin ratio was $89.4 \%$ and astaxanthin yield was up to $13.8 \mathrm{mg} / \mathrm{g} \mathrm{DCW}$ ) was achieved in 5-L fermenter. Our study not only provides new gene targets for astaxanthin biosynthesis, but also highlights the importance of the combined effects of metabolic engineering and mutagenesis on microbial overproduction of natural products.

\section{Results and discussion}

Enhancing astaxanthin yield and ratio by involving novel combination of CrtZ-CrtW

CrtZ and CrtW are widely found in bacteria, plants, archaebacteria and other organisms [23, 24]. The combination of these two enzymes is critical to astaxanthin production [6]. Our previous study further demonstrated that CrtW is more crucial to astaxanthin accumulation than CrtZ [25]. CrtW from Brevundimonas vesicularis DC263 (BDC263_CrtW) has been proved as a promising ketolase exhibiting general substrate diversity [25].
Although the combination of BDC263_CrtW and Asp_ CrtZ (CrtZ from Alcaligenes sp. strain PC-1) achieved better astaxanthin yield among our former thirty tested groups [25], canthaxanthin was still accumulated as the major intermediate in the fermentation product (Fig. 2a), thus improving the hydroxylation of canthaxanthin by CrtZ would be beneficial for further promoting astaxanthin yield and ratio here.

Fraser et al. [26] once compared the catalytic activity of E. coli-purified Aa_CrtZ (CrtZ from Agrobacterium aurantiacum) and Asp_CrtZ. As a result, Aa_CrtZ showed 2.6-fold enzyme activity of Asp_CrtZ with $\beta$-carotene as the substrate $(9.0-3.4 \mathrm{pmol} / \mathrm{h} / \mathrm{mg}$ protein) and 2.1-fold enzyme activity of Asp_CrtZ using canthaxanthin as the substrate $(60-28 \mathrm{pmol} / \mathrm{h} / \mathrm{mg}$ protein). Consistently, Aa_CrtZ achieved 68.8\% increase on zeaxanthin accumulation than Asp_CrtZ, when these two enzymes were individually expressed in our $\beta$-carotene producer SyBE_Sc118030 (Additional file 1: Figure S2). Therefore, in this study, the novel combination of CrtZ-CrtW (Aa_CrtZ-BDC263_CrtW) was adopted and expressed (Additional file 1: Figure S1) in our existing high $\beta$-carotene producer SyBE_Sc118030, gaining strain SyBE_Sc307001 (Table 2). Meanwhile, Real-Time PCR was conducted to investigate the transcriptional level of CrtZ and CrtW in strains SyBE_ Sc118060 and SyBE_Sc307001, and SyBE_Sc118060. Samples were harvested at $12 \mathrm{~h}$ (early phase) and $60 \mathrm{~h}$ (late phase) during the cultivation. As shown in Fig. 2a 


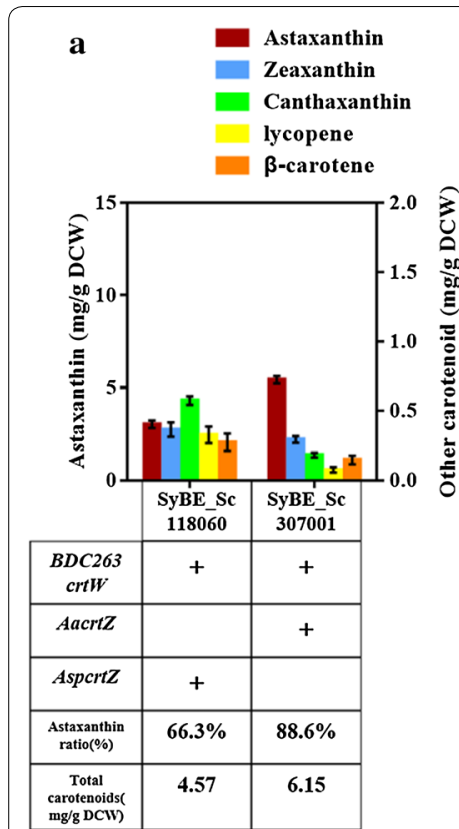

b

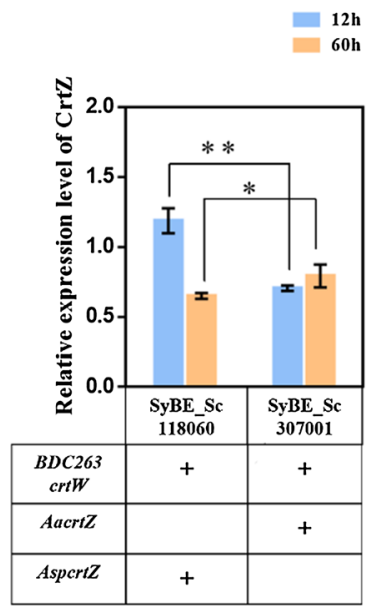

c

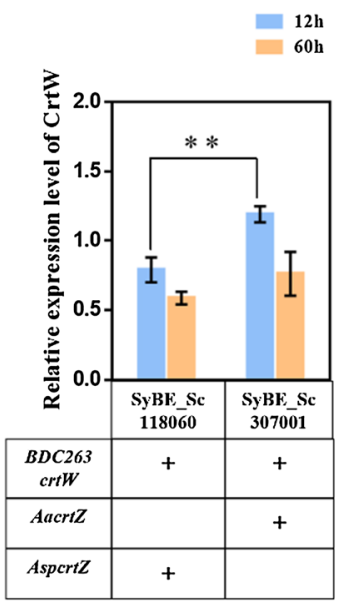

d

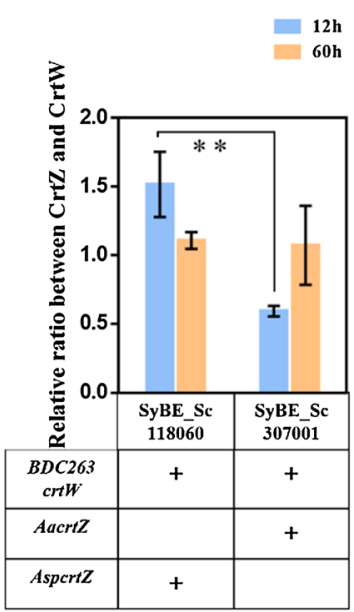

Fig. 2 The effect of new combination of CrtZ-CrtW on astaxanthin yield and ratio. a Carotenoids profiles in strain SyBE_SC118060 (with BDC263_ CrtW-Asp_CrtZ) and SyBE_Sc307001 (with BDC263_CrtW-Aa_CrtZ). Strains were cultured in YPD medium for $84 \mathrm{~h}$. The yield of astaxanthin and other carotenoid intermediates was analyzed by HPLC. "Astaxanthin yield" was determined as "the astaxanthin content in single cell" with unit as mg/g DCW; "astaxanthin ratio" was determined as "the astaxanthin content (mg/g DCW) to the total carotenoids content (mg/g DCW) in single cell" as \%. Other carotenoids indicated zeaxanthin, canthaxanthin, lycopene and $\beta$-carotene. b The relative transcription level of CrtZ. c The relative transcription level of $\mathrm{CrtW}$. $\mathbf{d}$ The relative ratio of $\mathrm{CrtZ}$ to $\mathrm{CrtW}$. Cells were harvested at $12 \mathrm{~h}$ (early phase) and $60 \mathrm{~h}$ (late phase). The relative transcription level for each gene was determined as $-\Delta C \mathrm{C}$, using gene ALG9 for normalization. The relative ratio of $\mathrm{CrtZ}$ to $\mathrm{CrtW}$ was calculated as $2^{-\Delta C \mathrm{t}(\mathrm{Cr} Z \mathrm{Z})} / 2^{-\Delta \mathrm{Ct}(\mathrm{Cr} \mathrm{tW})}$. All the error bars represent standard deviations calculated from three independent experiments. Significant levels of $t$-test: ${ }^{*} P<0.05,{ }^{* *} P<0.01$

and Additional file 1: Figure S2, the new combination achieved much less intermediate accumulation than the former one. To be noted, the accumulation of lycopene (peak IV, Additional file 1: Figure S2), $\beta$-carotene (peak V, Additional file 1: Figure S2) and canthaxanthin (peak III, Additional file 1: Figure S2) was reduced by 0.773-, 0.423- and 0.694-fold, respectively (Fig. 2a). The accumulation of canthaxanthin depends on the reaction competitiveness, including the efficiency of $\mathrm{CrtW}$ to generate canthaxanthin from $\beta$-carotene as well as the efficiency of $\mathrm{CrtZ}$ to convert canthaxanthin to astaxanthin. Less canthaxanthin accumulation in strain SyBE Sc307001 during time course might be caused by the increase in the relative transcriptional level of $\mathrm{Aa}$-CrtZ to $\mathrm{CrtW}$ as times went on (Fig. $2 \mathrm{~b}-\mathrm{d}$ ). Accordingly, the yield of astaxanthin (peak I, Additional file 1: Figure S2) was increased from $3.1 \mathrm{mg} / \mathrm{g}$ DCW to $5.5 \mathrm{mg} / \mathrm{g}$ DCW in strain SyBE_Sc307001 compared with strain SyBE_ Sc118060 (Fig. 2a). In the meanwhile, the astaxanthin ratio was also enhanced from 66.6 to $88.7 \%$ (Fig. 2a). Therefore, strain SyBE_Sc307001 was chosen as the starting strain to generate ARTP mutagenesis library.
Higher astaxanthin yield and total carotenoid production achieved by ARTP mutagenesis

To obtain cells with higher astaxanthin yield, strain SyBE_Sc307001 was submitted to ARTP for $30 \mathrm{~s}$ or $40 \mathrm{~s}$ individually and then grown on SD medium [27]. The death rate reached $82.6 \%$ and $86.8 \%$ under 30 -s and 40 -s treatments, respectively (Additional file 1: Figure S3). After radiation, seven strains exhibiting darker red pigments (SyBE_Sc2110M1-M7, Fig. 3 and Additional file 1: Figure S4) were visually picked up and then cultured to analyze their carotenoids compositions. As illustrated in Fig. 3a and Additional file 1: Figure S4a, all these mutants presented comparable cell growth to that of the control strain SyBE_Sc307001 in YPD medium [27]. In particular, astaxanthin yield in strain SyBE_Sc2110M3 was increased from $5.5 \mathrm{mg} / \mathrm{g}$ DCW to $10.1 \mathrm{mg} / \mathrm{g}$ DCW compared with the starting strain SyBE_Sc307001 (Fig. 3b), which was the highest astaxanthin yield at shake-flask level in S. cerevisiae to date (Table 1). Besides, more than 0.25 -fold improvements on total carotenoid accumulation were also observed in strains SyBE_Sc2110M1 and SyBE_Sc2110M3 (Fig. 3b). Therefore, genomic 
Table 2 S. cerevisiae strains used in this study

\begin{tabular}{|c|c|c|}
\hline Strains & Description & Sources \\
\hline BY4742 & 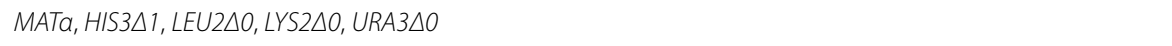 & [48] \\
\hline SyBE_Sc118030 & $\beta$-carotene-producing strain & [26] \\
\hline SyBE_Sc118060 & $\begin{array}{l}\text { Expression of BDC263_CrtW and Asp_CrtZ (ADH1t-Aa_crtZ-FBA1p-TDH3p-BDC263_crtW-TDH2t) in strain } \\
\text { SyBE_Sc118030 }\end{array}$ & [26] \\
\hline SyBE_Sc307001 & $\begin{array}{l}\text { Introducing plasmid BDC263_CrtW and Aa_CrtZ (ADH1t-Aa_crtZ-FBA1p-TDH3p-BDC263_crtW-TDH2t) } \\
\text { into strain SyBE_Sc118030 }\end{array}$ & This study \\
\hline SyBE_Sc307121 & Introducing plasmid pRS425K-Asp_CrtZ (FBA1p -Asp_crtZ-ADH1t) in strain SyBE_Sc118030 & This study \\
\hline SyBE_Sc307122 & Introducing plasmid pRS425K-Aa_CrtZ (FBA1p-Aa_crtZ-ADH1t) into strain SyBE_Sc118030 & This study \\
\hline SyBE_Sc2110M1 & ARTP mutant strain 1 of SyBE_Sc307001 & This study \\
\hline SyBE_Sc2110M2 & ARTP mutant strain 2 of SyBE_Sc307001 & This study \\
\hline SyBE_Sc2110M3 & ARTP mutant strain 3 of SyBE_Sc307001 & This study \\
\hline SyBE_Sc2110M4 & ARTP mutant strain 4 of SyBE_Sc307001 & This study \\
\hline SyBE_Sc2110M5 & ARTP mutant strain 5 of SyBE_Sc307001 & This study \\
\hline SyBE_Sc2110M6 & ARTP mutant strain 6 of SyBE_Sc307001 & This study \\
\hline SyBE_Sc2110M7 & ARTP mutant strain 7 of SyBE_Sc307001 & This study \\
\hline SyBE_Sc307104 & SyBE_Sc307001, $\triangle F L O 9:: K a n M X$ & This study \\
\hline SyBE_Sc307105 & SyBE_Sc307001, $\triangle Y$ YR410W-B::KanMX & This study \\
\hline SyBE_Sc307106 & SyBE_Sc307001, $\triangle Y B R 012 W-B:: K a n M X$ & This study \\
\hline SyBE_Sc307108 & SyBE_Sc307001, $\triangle D A N 4:: K a n M X$ & This study \\
\hline SyBE_Sc307109 & SyBE_Sc307001, $\triangle$ CSS1::KanMX & This study \\
\hline SyBE_Sc307120 & Integrating a Leu2 Marker into the $\mathrm{HO}$ site of strain BY4742 & {$[42]$} \\
\hline
\end{tabular}

comparison of strains SyBE_Sc2110M1, SyBE_Sc2110M3 and SyBE_Sc307001 would uncover potential gene targets contributing to higher astaxanthin yield and total carotenoid accumulation.

\section{Uncovering and validating gene targets contributing to higher astaxanthin yield by genomic and transcriptomics comparison}

As showed in Additional file 1: Figure S5, the copy numbers of the plasmid carrying BDC263_CrtW-Aa_CrtZ were unchanged among strains SyBE_Sc307001, SyBE_ Sc2110M1 and SyBE_Sc2110M3 which, therefore, did not contribute to the increase on astaxanthin yield by ARTP treatment. To identify the gene(s) with regard to such astaxanthin yield improvement, the whole genome of SyBE_Sc2110M1, SyBE_Sc2110M3 and SyBE_Sc307001 was re-sequenced and compared using SyBE_Sc307001 as the reference strain. As a result, there was no variation detected within gene $c r t Z$ and $c r t W$. To date, 61 variations affecting $22 \mathrm{CDS}$ and 89 variations affecting 45 intron/intergenic regions of strain SyBE_Sc2110M1 have been detected (Fig. 4a). Among these variations, there were total 27 sense mutations in CDS, containing 18 missenses, seven frame-shift mutations and two in frame insertions/deletions (Fig. 4a). Meanwhile, a total of 53 variations affecting 14 coding sequences (CDS), and 82 variations affecting 37 intron/intergenic regions of strain
SyBE_Sc2110M3 were counted (Fig. 4b). Excluding variations in intron/intergenic regions and synonymous substitutions, there was total of 18 sense variations in CDS, including 11 missenses, six frame-shift mutations and one in frame insertions/deletions (Fig. 4b).

Further comparison of CDS variations between SyBE Sc2110M1 and SyBE_Sc2110M3 only ascribed two variations solely to SyBE_Sc2110M3, i.e. one in YBR012W-B and one in $Y L R 410 \mathrm{~W}-B$ (Table 3 ). To verify whether their functions were related to astaxanthin yield, these two genes were individually knocked out in the control strain SyBE_Sc307001 (Additional file 1: Figure S6). Based on the annotation by Saccharomyces Genome Database (SGD, https://www.yeastgenome.org/) [28], YBR012W-B and $Y L R 410 W-B$ happened to be both involved in retrotransposon elements (Table 3 ). However, only deletion of $Y B R 012 W-B$ can significantly increase astaxanthin yield, while loss of $Y L R 410 \mathrm{~W}-B$ appeared to have no effect on astaxanthin yield (Fig. 5a). Retrotransposon can trigger chromosomal rearrangements and bring global perturbation on transcriptional profiles [29]. So far, it is difficult to interpret the different effect of $Y B R 012 \mathrm{~W}-\mathrm{B}$ and $Y L R 410 W-B$ on astaxanthin accumulation. Further transcriptome analysis might provide some clues for related underlying molecular mechanisms in future study.

Mutations in SyBE_Sc2110M1 and SyBE_Sc2110M3 can boost total carotenoid accumulation (by more than 

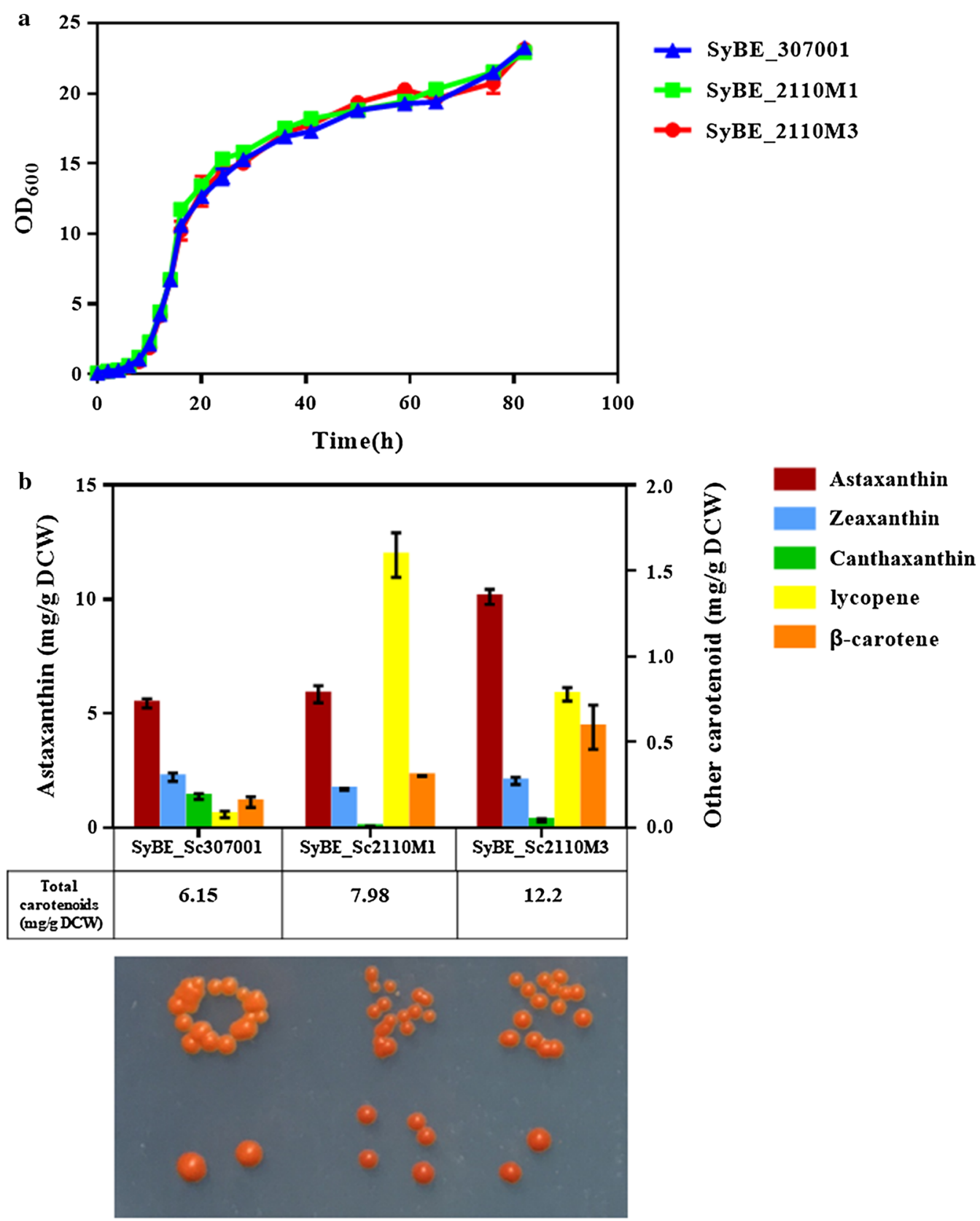

Fig. 3 Cell growth and astaxanthin yield of ARTP mutants. Growth curve $\mathbf{a}$ and carotenoid profile $\mathbf{b}$ of strain SyBE_Sc307001 and its ARTP mutagenesis strains (SyBE_SC2110M1 and SyBE_SC2110M3) which achieved higher total carotenoids accumulation in YPD medium than the parent strain. A photograph was attached bellow the bar chart to illustrate visual color of the related strains. The error bars represent standard deviations calculated from duplicate experiments. "Astaxanthin yield" was determined as "the astaxanthin content in single cell" with unit as mg/g DCW. Other carotenoids indicated zeaxanthin, canthaxanthin, lycopene and $\beta$-carotene

0.25 -fold) (Fig. 3b). As shown in Table 3, variations in genes FLO9, CSS1 and DAN4 occurred in high frequency within both SyBE_Sc2110M1 and SyBE_Sc2110M3. Thus, it was assuming that these genes might be crucial to total carotenoid accumulation, and even astaxanthin yield. To support this hypothesis, FLO9, CSS1 and DAN4 were also individually knocked out in the control strain SyBE_Sc307001 (Additional file 1: Figure S6). As illustrated in Fig. 5a, compared to the control strain, neither astaxanthin yield nor total carotenoid accumulation was significantly enhanced by single deletion of FLO9. Nevertheless, compared to the control strain, individual 


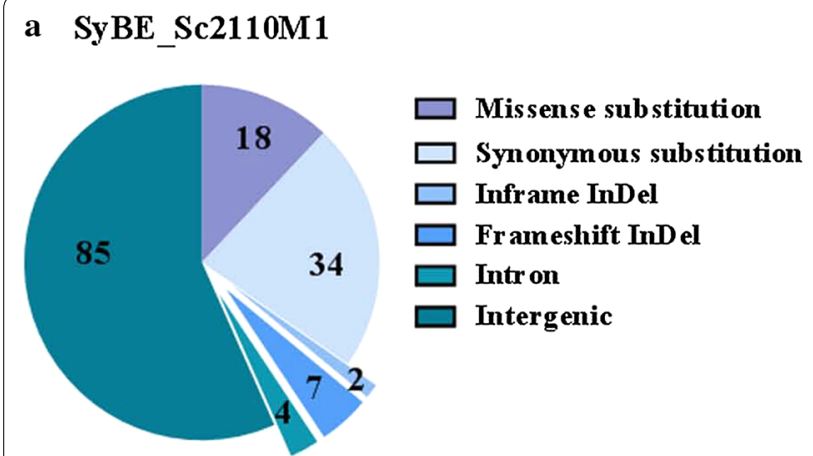

b SyBE_Sc2110M3

Fig. 4 The variations in selected ARTP mutants with respect to strain SyBE_Sc307001. Variations distribution of all the variations in strain SyBE_ Sc2110M1 (a) and SyBE_Sc2110M3 (b) with respect to strain SyBE_Sc307001

deletion of CSS1 or DAN4 enhanced astaxanthin yield by 0.596 - and 0.363 -fold, respectively (Fig. $5 \mathrm{a}$ ). Notably, $\triangle C S S 1$ achieved $88.9 \%$ of the astaxanthin yield of ARTPmutant SyBE_Sc2110M3 (Fig. 5a), indicating CSS1 played a more critical role in astaxanthin biosynthesis than DAN4.

In SGD, CSS1 is annotated as a putative glucan alpha1,4-glucosidase. Knockout of its homolog ISC1 (inositol phosphosphingolipid phospholipase C) in S. cerevisiae was found to accumulate more sphingolipids in cell membrane [30]. Consequently, RNA-seq analysis was applied to investigate the transcriptional effect of $\triangle C S S 1$ by comparing the control strain SyBE_Sc307001 with CSS1 knocked-out strain SyBE_Sc307109. Samples were taken at $12 \mathrm{~h}$ (early phase) and $60 \mathrm{~h}$ (late phase). As a result, when CSS1 was lost, genes associated with phospholipid metabolism showed no significant transcriptional difference. However, most of genes involved in biosynthesis of ergosterol (ERG1, ERG11, ERG25, ERG27, ERG6, $E R G 3, E R G 5$ and ERG4) were found to be upregulated in late phase (Fig. 6a), suggesting $\triangle C S S 1$ might enhance the intracellular sterol levels. Sterols are key components of the cytomembrane and are associated with cell tolerance to hydrophobic molecules (such as D-limonene) [31]. As reported, carotenoids tend to accumulate in membrane system [32], and membrane engineering via altering cell membrane composition has been proved to be a promising strategy to enhance the accumulation of desired hydrophobic products [33, 34]. Therefore, the improvement on astaxanthin yield by $\triangle$ CSS1 is probably due to its biofunction involving in cell membrane. Meanwhile, the RNA-seq data also illustrated that majority of genes involved in TCA cycle (PCK1, LDP1, CIT2, ACO1, IDH1, IDP1, KGD1/2, LPD1, SDH4, FUM1, MDH3) were found to be downregulated in early phase (Fig. 6b), which might explain the cell growth-deficient problems observed in strains with $\triangle C S S 1$ in both YPD medium
(Fig. 5b) and SD medium (Additional file 1: Figure S7). As demonstrated in Fig. 5 and Additional file 1: Figure $\mathrm{S} 7$, the higher astaxanthin production was caused by the combined effects of gene deletion (such as $\triangle$ CSS1 and $\triangle Y B R 012 W-B)$ and reduction in dry cell weight. Since strain SyBE_Sc2110M3, which carried all the gene variations, demonstrated comparable cell growth with the control strain (Fig. 5b and Additional file 1: Figure S7), further gene deletion in different combination such as deletion of CSS1/DAN4 and CSS1/FLO9 would be carried to show variations in cell growth.

\section{Astaxanthin overproduction by fed-batch fermentation}

Before bioprocess optimization, the genetic stability of strain SyBE_Sc2110M3 was investigated. As shown in Additional file 1: Figure S8, the astaxanthin yield in strain SyBE_Sc2110M3 was stable after passing six generations in SD medium. Thus, strain SyBE_Sc2110M3 was selected for fed-batch fermentation to further promote the astaxanthin production. To achieve high cell density fermentation for high astaxanthin accumulation, carbon restriction strategy was applied according to Wang et al. [25]. Fed-batch fermentation was conducted in 5-L bioreactor and the glucose feeding was strictly controlled below $2 \mathrm{~g} / \mathrm{L}$. Finally, the total volume of the endpoint fermentation in 5-L bioreactor was almost $3.9 \mathrm{~L}$. As shown in Fig. 7a, after the initial glucose was exhausted at $12 \mathrm{~h}$, astaxanthin yield entered a sharply increasing period until the yield reached maximal value $(13.8 \mathrm{mg} / \mathrm{g} \mathrm{DCW})$ at $68 \mathrm{~h}$. This astaxanthin yield obtained in 5-L bioreactor is higher than that at flask-flash level (10.1 mg/g DCW). In the meantime, the main intermediates (lycopene, $\beta$-carotene, canthaxanthin and zeaxanthin) were gradually converted and converged to the targeted product astaxanthin. The intermediate conversion fastigium was observed in the period from 12 to $47 \mathrm{~h}$, and the astaxanthin ratio was sharply enhanced from 54.2 to $83.8 \%$ 
Table 3 CDS variations in SyBE_SC2110M1 and SyBE_SC2110M3 with respect to SyBE_Sc307001

\begin{tabular}{|c|c|c|c|}
\hline Chromosome & Gene & Position and mutate & Function \\
\hline \multicolumn{4}{|c|}{ Specific to SyBE_Sc2110M1 } \\
\hline \multirow[t]{3}{*}{ ChrlV } & HKR1 & 1308011: $V<->A^{a}$ & Zinc finger family member \\
\hline & & 1308047: $V<->A^{a}$ & \\
\hline & URC2 & 1482052: $Y<->C^{a}$ & Putative transcription factor \\
\hline \multirow[t]{2}{*}{ ChrVII } & PDR1 & 470224: $C<->F^{a}$ & Transcription factor \\
\hline & GTO1 & 797427: $K<->Q^{a}$ & Glutathione transferase \\
\hline ChrXII & UBR2 & 188380: $\mathrm{Q}<->\mathrm{H}^{\mathrm{a}}$ & Cytoplasmic ubiquitin-protein ligase \\
\hline \multirow[t]{3}{*}{ ChrXIII } & YMR317W & 908196: $W<->S^{a}$ & Hypothetical protein \\
\hline & & 908198: $A<->S^{a}$ & \\
\hline & YML084W & 99616D: $\mathrm{AT}^{\mathrm{b}}$ & Hypothetical protein \\
\hline \multirow[t]{2}{*}{ ChrXV } & NDD1 & 1036169D:TTGC & Transcriptional activator \\
\hline & YOL166C & 1109D: TA ${ }^{b}$ & Hypothetical protein \\
\hline ChrXVl & RRG8 & 765953: $\mathrm{N}<->\mathrm{K}^{\mathrm{a}}$ & Hypothetical protein \\
\hline \multicolumn{4}{|c|}{ Specific to SyBE_Sc2110M3 } \\
\hline Chrll & YBR012W-B & 263145D: $C^{b}$ & Retrotransposon TYA Gag \\
\hline ChrXII & YLR410W-B & 944238: $Q<->K^{a}$ & Retrotransposon TYA Gag and TYB Pol \\
\hline \multicolumn{4}{|l|}{ Commonalities } \\
\hline \multirow[t]{3}{*}{ Chrl } & FLO9 & $27080: V<->\left.\right|^{a}$ & Mannose-binding lectin \\
\hline & & $25872: 1<->M^{a}$ & \\
\hline & & 26144: $M<->L^{a}$ & \\
\hline Chrll & YBL100W-A & 30754: $\mathrm{D}<->\mathrm{N}^{\mathrm{a}}$ & Retrotransposon TYA Gag \\
\hline Chrlll & TAF2 & 2044361: CTTCCTCTTCC ${ }^{b}$ & RNA polymerase II transcription initiation \\
\hline ChrlV & YDR340W & 11509131: G & Hypothetical ORF \\
\hline \multirow[t]{2}{*}{ ChrVIII } & YHLO50C & 1748: $\mathrm{N}<->\mathrm{S}^{\mathrm{a}}$ & Hypothetical ORF \\
\hline & YHLO4IW & 17546l: $T^{\mathrm{b}}$ & Hypothetical ORF \\
\hline \multirow[t]{2}{*}{ ChrlX } & CSS1 & 25586: $D<->G^{a}$ & Hypothetical ORF \\
\hline & & 25418: $D<->G^{a}$ & \\
\hline \multirow[t]{3}{*}{ ChrX } & DAN4 & 715095: $P<->S^{a}$ & Cell wall mannoprotein \\
\hline & & 715122: $P<->S^{a}$ & \\
\hline & YJR023C & 470178: D: ATA ${ }^{\mathrm{C}}$ & Protein required for cell viability \\
\hline ChrXI & YKL2225W & $4581: A^{b}$ & Hypothetical ORF \\
\hline ChrXV & YOR192C-B & 708819: $A<->G^{a}$ & Retrotransposon TYA Gag \\
\hline ChrXIV & YNL338W & 6637D: $A C^{b}$ & Hypothetical ORF \\
\hline ChrXVI & DPB2 & 896899: $F<->Y^{a}$ & DNA polymerase epsilon \\
\hline
\end{tabular}

a Missense substitutions, numbers indicated the mutagenesis sites in chromosome, capital letters indicated the corresponding mutations on amino acids

b Frame-shift mutations, numbers indicate the mutagenesis sites in chromosome, " $\mathrm{D}$ " is for deletion, "I" is for insertion, capital letters after ":" indicates the deleted or inserted base(s)

c In frame insertions/deletions, numbers indicate the mutagenesis sites in chromosome, " $\mathrm{D}$ " is for deletion, capital letters after ":" indicates the deleted or inserted base(s)

(Fig. 7b). It was also noted that the cell density increased faster than astaxanthin titer after $68 \mathrm{~h}$, leading to a dramatic decrease in astaxanthin yield. Such threshold indicated we should optimize the feeding strategies to balance the cell growth and target product production in future work. Eventually, a titer of $217.9 \mathrm{mg} / \mathrm{L}$ astaxanthin was obtained after $140 \mathrm{~h}$ cultivation (Fig. 7a). At that point, the astaxanthin ratio reached to $89.4 \%$ (Fig. $7 \mathrm{~b}$ ). Indeed, the titer was not satisfactory for large-scale commercialization. Considering the cell stress caused by astaxanthin accumulation [35], exploring inducible promoters (such as GAL promoters) to control the expression of heterologous genes could achieve high cell-density fermentation by decoupling cell growth with product accumulation, which could significantly shorten the cell growth period and concentrate optimization for astaxanthin yield enhancement by corresponding feeding strategy. To figure out potential biomarkers by comparing 

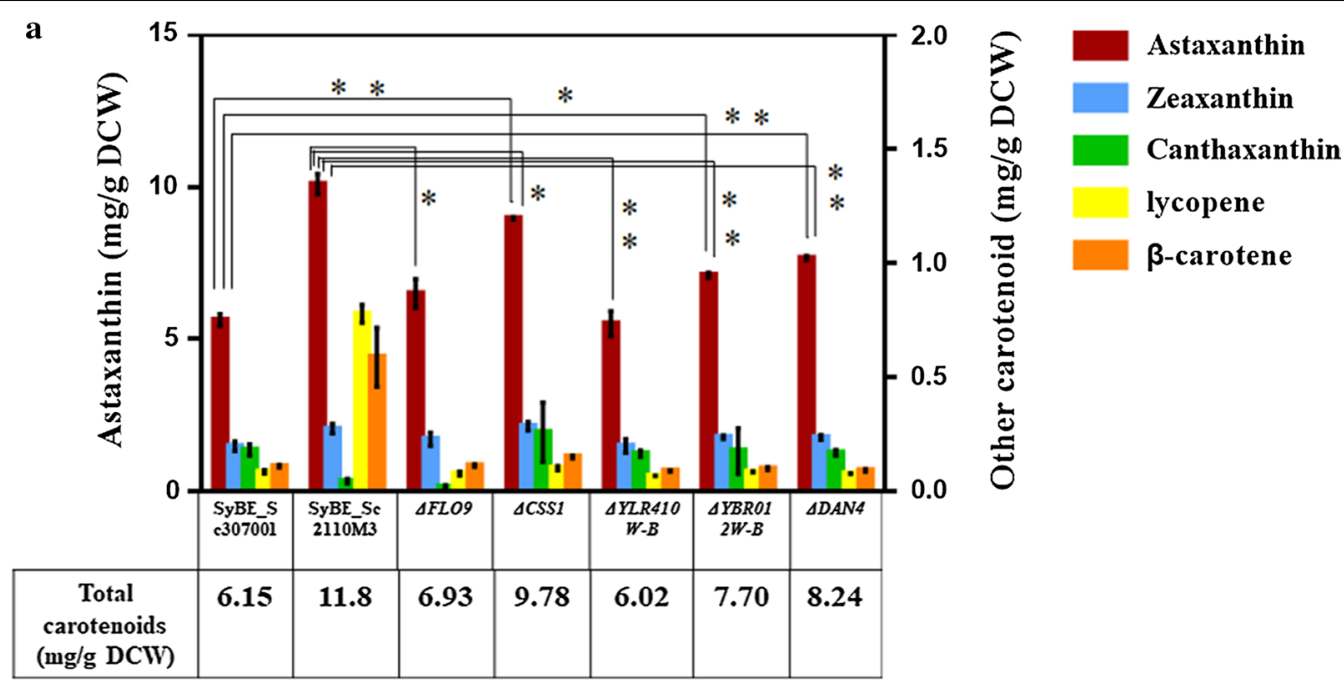

b

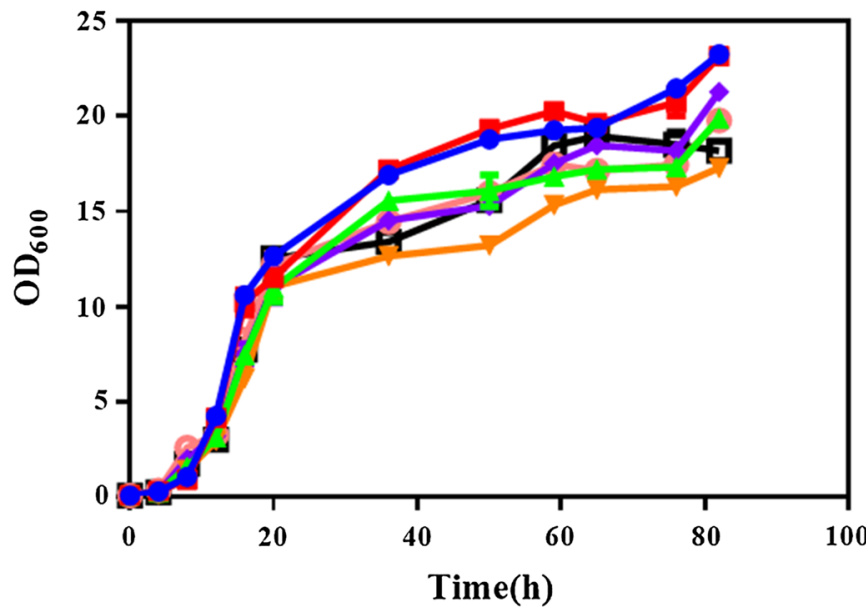

$\sim$ SyBE_307001

$\rightarrow$ SyBE_2110M3

$\because \triangle F L O 9$

$\rightarrow \triangle C S S I$

$\sim \triangle Y L R 410 W-B$

- $-\triangle Y B R 012 W-B$

\# $\triangle D A N 4$

Fig. 5 The effect of CDS variations involved in astaxanthin biosynthesis. Carotenoid profile (a) and growth curve (b) of SyBE_SC307001, SyBE_ Sc2110M3 and gene knocked-out strains in YPD medium. These gene-deleted strains were generated from strain SyBE_Sc307001 by individual loss of genes FLO9, CSS1, YLR41OW-B, YBRO12W-B and DAN4, respectively. "Astaxanthin yield" was determined as "the astaxanthin content in single cell" with unit $\mathrm{mg} / \mathrm{g}$ DCW. Other carotenoids indicated zeaxanthin, canthaxanthin, lycopene and $\beta$-carotene. The error bars represent standard deviation calculated from triplicate experiments. Significance levels of $t$-test were determined as "** is for $P<0.05$ and "**" is for $P<0.01$

different astaxanthin accumulation period by metabolomics analysis could also be a powerful source to guide media and feeding strategy optimization [36].

\section{Conclusions}

A comprehensive approach integrating heterologous module engineering and mutagenesis by ARTP was employed to promote astaxanthin yield to $10.1 \mathrm{mg} / \mathrm{g}$ DCW, which is the highest reported yield at shake-flask level in S. cerevisiae so far. Astaxanthin yield and ratio was significantly enhanced by involving new combination of CrtZ and CrtW (Aa_CrtZ-BDC263_CrtW).
Three underlying gene targets (CSS1, YBRO12W-B and $D A N 4)$ associated with astaxanthin biosynthesis were first uncovered by comparative genomics analysis. Notably, deletion of CSS1 can recover $75.6 \%$ improvement on astaxanthin yield by ARTP, indicating CSS1 is a key molecular target for astaxanthin accumulation. RNAseq analysis indicates that CSS1 deletion effectively upregulates membrane composition synthesis. Eventually, $217.9 \mathrm{mg} / \mathrm{L}$ astaxanthin (astaxanthin ratio was $89.4 \%$ and astaxanthin yield was up to $13.8 \mathrm{mg} / \mathrm{g}$ DCW) was obtained in 5-L fermentor without any addition of inducers. This combinatorial strategy might be also applicable 


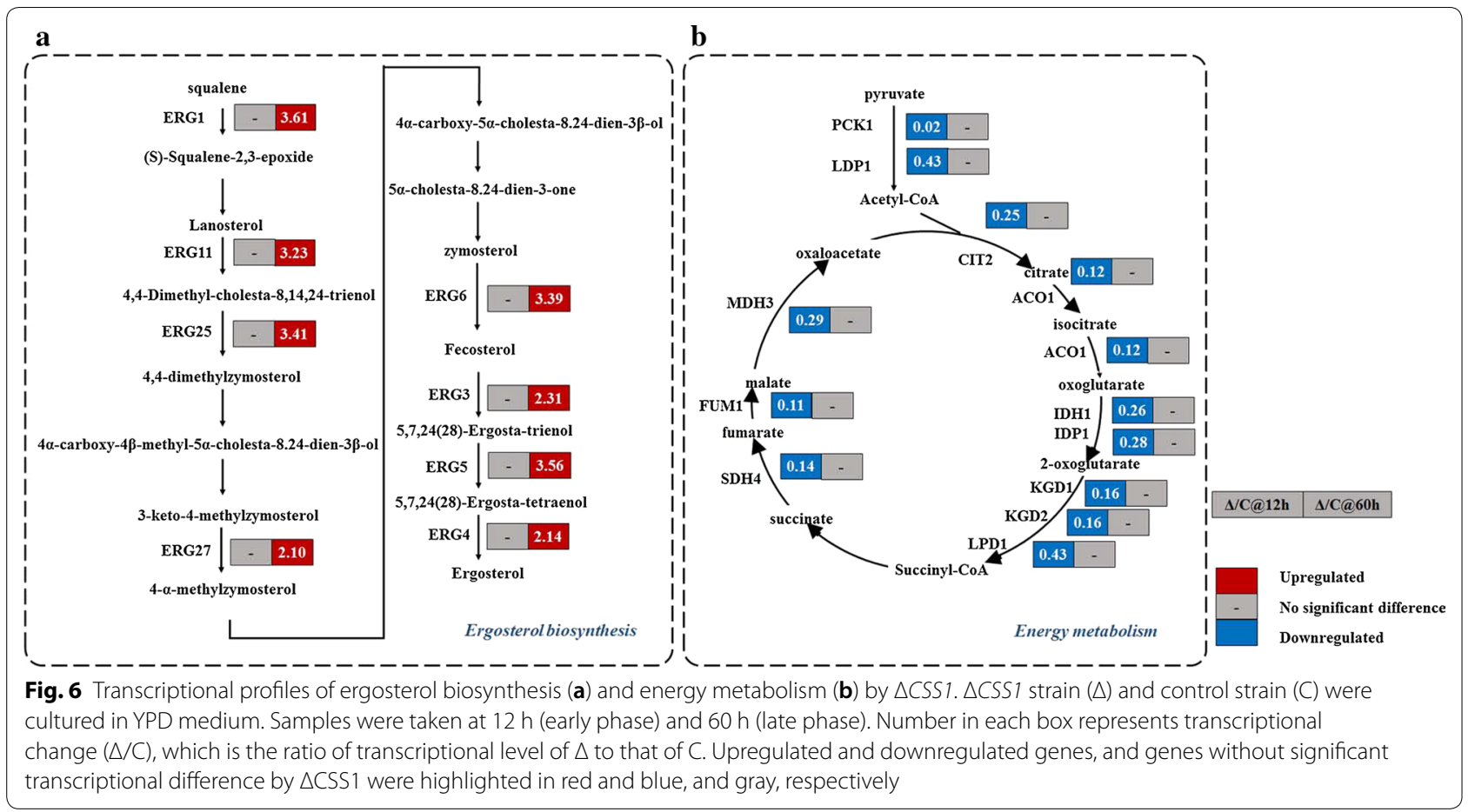

for biosynthesis of other value-added products, especially colored metabolites. And either increasing the round of mutagenesis or iterative operation of metabolic engineering and mutagenesis by screening in several rounds would be very promising for future optimization. In terms of current system, exploring process optimization (i.e. medium optimization and feeding strategy testing) to balance the carbon flux towards biomass built-up and astaxanthin biosynthesis is also very helpful for further improvement, in which more on-line parameter feedback control strategy guided by off-gas analysis will be very essential [37].

\section{Methods}

\section{Strains and cultivation}

All the strains used in this study are described in Table 2. E. coli DH5 $\alpha$ was used for plasmid construction and replication. E. coli strains were cultured at $37{ }^{\circ} \mathrm{C}$ in LuriaBertani (LB) complete medium. $50 \mu \mathrm{g} / \mathrm{mL}$ kanamycin or $100 \mu \mathrm{g} / \mathrm{mL}$ ampicillin was added into the medium for selection. In the meanwhile, yeast cells were cultured at $30{ }^{\circ} \mathrm{C}$ in YPD medium [27]. For astaxanthin fermentation in shake-flask, a single yeast colony was inoculated into $3 \mathrm{~mL} \mathrm{SD} \mathrm{medium} \mathrm{[27]} \mathrm{and} \mathrm{grown} \mathrm{at} 30{ }^{\circ} \mathrm{C}$ until $\mathrm{OD}_{600} \approx 8.0$. Then the preculture was inoculated into $3 \mathrm{~mL}$ fresh SD medium with an initial $\mathrm{OD}_{600}$ of 0.2 for further $14 \mathrm{~h}$ cultivation (to $\mathrm{OD}_{600} \approx 6.0$ ). After that, the seed culture was transferred into $50 \mathrm{~mL}$ fresh YPD medium for $84 \mathrm{~h}$ at an initial $\mathrm{OD}_{600}$ of 0.1 and grew until harvest.

\section{Construction of plasmids and strains}

All the plasmids used in this study are listed in Additional file 1: Table S1. Genes encoding CrtW from $B$. vesicularis DC263 (BDC263_CrtW) and CrtZ from $A$. aurantiacum (Aa_CrtZ) were recovered by BsaI digestion from plasmids pUC57-Simple-14 and pUC57-Simple-01, respectively. These two plasmids were generated in our previous work via cloning genes BDC263_crtW and Aa_crtZ (codon optimized and synthesized by Genscript Inc.) into plasmid pUC57-simple [25]. Construction of BDC263_CrtW-Aa_CrtZ expression cassette plasmid (pRS425K-BDC263_CrtW-Aa_CrtZ) (Additional file 1: Figure S1a) and CrtZ expression cassette plasmid (pRS425K-Asp_CrtZ/pRS425K-Aa_CrtZ) (Additional file 1: Figure S2a) followed the procedure described in our previous work [25]. The constructed product was verified by PstI/BamHI digestion and DNA-sequencing. Transformation of the particular plasmid into strain SyBE_Sc118030 was conducted by LiAc/SS carrier DNA/ PEG method [38]. The engineered yeast strains were selected on SD medium with the appropriate amino acid drop out mix supplementation.

PCR-mediated gene disruptions were applied with the primers provided in Additional file 1: Table S2. Yeast homologous arms and KanMX marker were amplified from the genomic DNA of S. cerevisiae SyBE_Sc118030 


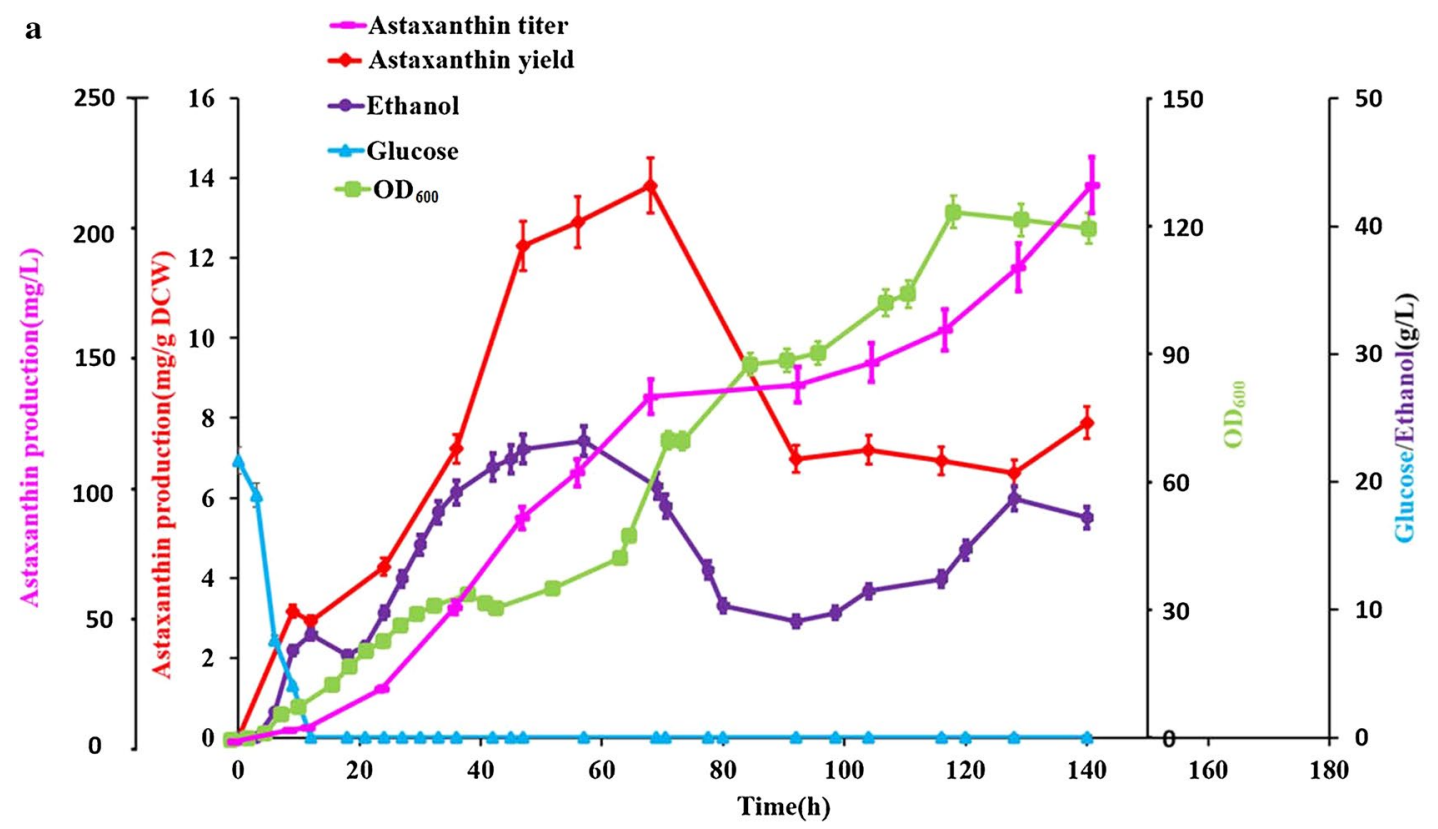

b

9 h

$12 \mathrm{~h}$

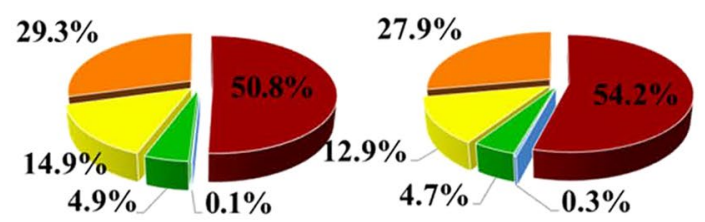

$68 \mathrm{~h}$

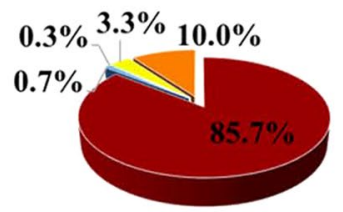

$92 \mathrm{~h}$

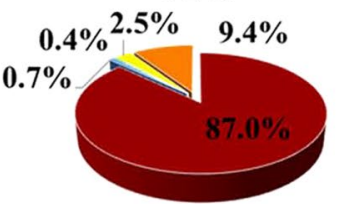

$24 \mathrm{~h}$

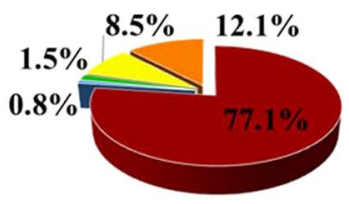

$47 \mathrm{~h}$

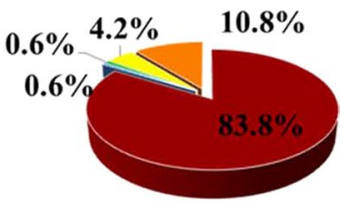

$\boldsymbol{\beta}$-corotene

lycopene Canxanthathin

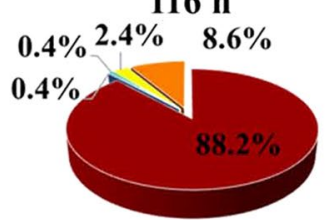

$140 \mathrm{~h}$

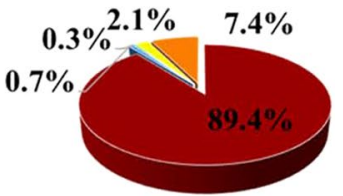

Zeaxanthin

Astaxanthin

Fig. 7 Astaxanthin production in fed-batch fermentation. a Profile of astaxanthin yield (pink), astaxanthin titer (red), glucose (blue), ethanol (purple) and cell density (green) during fermentation with strain SyBE_Sc2110M3. The error bars represent standard deviation calculated from duplicate experiments. "astaxanthin yield" was determined as "the astaxanthin content in single cell" with unit mg/g DCW; "astaxanthin titer" was determined as "the astaxanthin concentration in bioreactor" with unit mg/L. b The trend of carotenoid composition (the content (mg/g DCW) of a particular carotenoid to the total carotenoid content (mg/g DCW) in single cell, resented in \%) in fermentation product. Astaxanthin, zeaxanthin, canthaxanthin, lycopene and $\beta$-carotene were labeled by red, blue, green, yellow and orange, respectively

and plasmid bYW0182, respectively. The gene knockout cassette (left homologous arm-KanMX-right homologous arm) was assembled by overlap extension PCR (OE-PCR). Then the product was purified and transformed into strain SyBE_Sc307001. The gene-deleted strains were verified by PCR with the primers listed in Additional file 1: Table S2. One pair of the verified primers (I-F/I-R, Additional file 1: Figure S6a) was located upstream of the left homologous arm and downstream of the right homologous arm of the targeted gene; while another pair of the verified primers (II-F/II-R, Additional file 1: Figure S6a) was located inside of the targeted gene. Primers I-F/I-R are able to amplify a clear band in the DNA poles for gene 
knocked-out stains (i.e. 2960 bp for $\triangle F L O 9,2089$ bp for $\triangle C S S 1,2296$ bp for $\triangle D A N 4,3277$ bp $\triangle Y B R 012 W-$ $B, 2745$ bp for $\triangle Y L R 410 W-B$ ) while primers II-F/II-R are able to amplify a clear band only in the DNA poles for the parent stain (i.e. $2012 \mathrm{bp}$ for $F L O 9,1823 \mathrm{bp}$ for CSS1, 2168 bp for DAN4, 2054 bp for $Y B R 012 W-B$, 1966 bp for $Y L R 410 W-B)$.

\section{Construction of ARTP mutagenesis library}

After growing in SD medium for $30 \mathrm{~h}$, the culture was transferred to fresh SD medium until $\mathrm{OD}_{600}$ approaches 1.0. Then $10 \mu \mathrm{L}$ of the culture was spread on a sterile iron plate to be irradiated by ARTP with a power of $100 \mathrm{~W}$ and a gas flow of 10 SLM. The distance between the plasma torch nozzle exit and the sample plate was $2 \mathrm{~mm}$. Since it requires a death rate of $80 \%-90 \%$ to achieve effective mutation [39, 40], cells were submitted to ARTP for 10, $15,20,30,40,50,60,80,100,120$ and $150 \mathrm{~s}$, respectively. After that, all the treated cells were washed from the plate and further grown on SD agar plates for $48 \mathrm{~h}$. It was found that only 30-s and 40-s treatments could obtain a death rate between 80 and $90 \%$ (i.e. $82.6 \%$ for $30 \mathrm{~s}$ and $86.8 \%$ for $40 \mathrm{~s}$, Additional file 1: Figure S3). Thus, our ARTP mutagenesis library covered the cells treated by ARTP for both $30 \mathrm{~s}$ and $40 \mathrm{~s}$. After obtaining ARTP mutagenesis library, visual color screening [25] could be performed to choose the candidates with dark red color for higher astaxanthin yield. The genetic stability assay was conducted in SD medium. The candidate strain(s) was sub-cultured for six generations. And the astaxanthin yield was monitored accordingly.

\section{Quantitative real-time PCR}

Quantitative real-time PCR (qPCR) was applied to quantify copy numbers of plasmid or to measure the transcriptional levels of genes in engineered strains. The copy number assay was referred to Jia et al. [41]. Yeast genomic DNA was employed as the template in qPCR analysis. Strain SyBE_Sc307120 with single copy LEU2 marker was used as the reference strain. The copy numbers were determined by comparing the $\mathrm{Ct}$ values of $L E U 2$ and the reference gene $A L G 9$ using the $2^{-\Delta \Delta \mathrm{Ct}}$ method [42]. For transcription level analysis, strains were sampled at (early phase) and $60 \mathrm{~h}$ (late phase), respectively. The method of total RNA extraction, reverse transcription and qPCR procedure was as same as Wang et al. [25], except gene ALG9 was adopted for normalization. The relative transcription level for $\mathrm{CrtZ}$ and $\mathrm{CrtW}$ was determined as $-\Delta \mathrm{Ct}$ [43], using gene ALG9 for normalization. The relative ratio of $\mathrm{CrtZ}$ to $\mathrm{CrtW}$ was calculated as $2^{-\Delta \mathrm{Ct}(\mathrm{CrtZ})} / 2^{-\Delta \mathrm{Ct}(\mathrm{CrtW})}$.
Genome re-sequencing and comparative genome analysis Whole genome re-sequencing of strains SyBE_Sc307001, SyBE_Sc2110M1 and SyBE_Sc2110M3 was carried out by Beijing Novogene Bioinformatics Technology Co., Ltd. Yeast cultures were grown to saturation, and then genomic DNA was isolated to prepare amplicon-free libraries. Paired-end sequencing for all libraries was performed on the Illumina HiSeq2000 platform. Burrows-Wheeler alignment (BWA) [44] was used to align the paired-end reads against the sequences of $S$. cerevisiae S288C (Accession No. NC_001136) and the heterologous modules provided by our group. SAMtools [45] was used to detect the individual SNPs (single nucleotide polymorphisms) and Indels (insertion and deletion of fragments $<50 \mathrm{bp}$ ), as well as analyze the variation of SNPs/InDels between strains SyBE_Sc2110M1/M3 and SyBE_Sc307001.

\section{RNA-sequencing analysis}

Cells were harvested from cultivation at $12 \mathrm{~h}$ (early phase) and $60 \mathrm{~h}$ (late phase), respectively. Total RNA was isolated following the NEB Next Ultra ${ }^{\mathrm{TM}}$ RNA protocol and using NEB Next Poly(A) mRNA Magnetic Isolation Module (NEB) according to the manufacturer's instructions and was quantified and qualified by Agilent 2100 Bioanalyzer. Sequencing was processed by Genewiz Inc. on Illumina HiSeq2500 platform. Image analysis and base calling were conducted by HiSeq control software on the HiSeq instrument. Htseq software was used to normalize the data using RPKM (Reads per Kilo bases per Million reads)-based normalization algorithm [46]. Differentially expressed genes were identified by DESeq2 software [47] with $\log 2$ foldchange $>1.0$ and a corrected $P$-value $<0.05$. $\mathrm{R}$ software was used for hierarchical clustering analysis. RNA-seq data were calculated from two biological replicates. The Saccharomyces genome database (SGD) [28] was used to gain gene information.

\section{Fed-batch fermentation}

Fed-batch fermentation was conducted in a 5-L bioreactor (T\&J Bioengineering Co., Ltd, Shanghai, China) under glucose restriction strategy. $100 \mu \mathrm{L}$ glycerolstock of strain SyBE_Sc2110M3 was inoculated into $50 \mathrm{~mL} \mathrm{SD}$ medium and cultured at $30{ }^{\circ} \mathrm{C}, 250 \mathrm{rpm}$ for overnight growth. Then the preculture was transferred to $200 \mathrm{~mL}$ fresh SD medium and grew until entering mid-exponential phase. $10 \%(\mathrm{v} / \mathrm{v})$ seed culture was transferred to $1.8 \mathrm{~L}$ YPD medium (containing $20 \mathrm{~g} / \mathrm{L}$ glucose) in 5-L bioreactor. The fermentation was carried out at $30{ }^{\circ} \mathrm{C}$. The $\mathrm{pH}$ was automatically controlled at 5.8 with $6 \mathrm{M}$ sodium hydroxide. And the dissolved oxygen was kept at $30 \%$ by agitation cascade from 400 
to $800 \mathrm{rpm}$, while the air flow was set at $1.5 \mathrm{vvm}$. The glucose concentration was monitored every $3 \mathrm{~h}$. And after the initial glucose was depleted, an appropriate volume of $500 \mathrm{~g} / \mathrm{L}$ glucose solution was supplemented to maintain the glucose concentration less than $2 \mathrm{~g} / \mathrm{L}$. $30 \mathrm{~g}$ yeast extract was added into the bioreactor every $12 \mathrm{~h}$ by adding $500 \mathrm{~g} / \mathrm{L}$ yeast extract stock solution. Duplicate samples were collected to determine the cell density, glucose concentration, ethanol concentration and astaxanthin production.

\section{Determination of glucose, ethanol and carotenoids}

The concentrations of glucose and ethanol in the culture were analyzed by a reverse-phase high-performance liquid chromatography (HPLC) system consisting of a Waters 1515 pump (Milford, MA, USA) and a Waters 2414 refractive index detector. The samples were separated on an Aminex HPX-87H carbohydrate analysis column (Bio-Rad, Hercules, CA, USA) at $65{ }^{\circ} \mathrm{C}$ using $5 \mathrm{mM}$ sulfuric acid as the mobile phase with a flow rate of $0.6 \mathrm{~mL} / \mathrm{min}$.

Carotenoids were extracted from the $\mathrm{HCl}$-heattreated cells with acetone according to Zhou et al. [11] and Wang et al. [25]. To be specific, cells from $2 \mathrm{~mL}$ culture were collected and washed with distilled water. After that, the cells were suspended in $1 \mathrm{~mL} 3 \mathrm{M} \mathrm{HCl}$ for 5 min boiling at $100{ }^{\circ} \mathrm{C}$ in sealed tubes and then quickly chilling on ice. The cell debris were harvested, washed with distilled water twice and resuspended in $0.5 \mathrm{~mL}$ of acetone containing $1 \%(\mathrm{w} / \mathrm{v})$ butylated hydroxytoluene. The above mixture was vortexed for $20 \mathrm{~min}$ and incubated at $30{ }^{\circ} \mathrm{C}$ for $10 \mathrm{~min}$. After centrifugation at 12,000 rpm for $5 \mathrm{~min}$, the acetone phase was filtered by $0.22-\mu \mathrm{m}$ membrane. The whole process was performed in darkness. And all centrifugal tubes used for astaxanthin extraction were covered with aluminum foil to avoid exposure to light. The extracted products were analyzed by HPLC (Waterse2695, Waters Corp., USA) equipped with a BDS HYPERSIL C18 column (150 mm $\times 4.6 \mathrm{~mm}, 5 \mu \mathrm{m}$, Thermo Scientific) and a UV/ VIS detector (Waters 2489) at $470 \mathrm{~nm}$ [25]. Carotenoids standards (astaxanthin, zeaxanthin, canthaxanthin, lycopene and $\beta$-carotene) were purchased from Sigma (Sigma-Aldrich, MO, USA) for qualitative and quantitative analysis. To describe astaxanthin productivity, "the astaxanthin content in single cell" was determined as "astaxanthin yield" with unit $\mathrm{mg} / \mathrm{g}$ DCW; "the astaxanthin concentration in bioreactor" was determined as "astaxanthin titer" with unit $\mathrm{mg} / \mathrm{L}$; "the astaxanthin content $(\mathrm{mg} / \mathrm{g} \mathrm{DCW})$ to the total carotenoids content (mg/g DCW) in single cell" was determined as "astaxanthin ratio" in \%.

\section{Statistical analysis}

All the statistical analysis was conducted by SPSS 19.0. The level of significance was set at $P<0.05$. All the error bars represented at least independent duplicate experiments.

\section{Additional file}

Additional file 1: Table S1. Plasmids used in this study. Table S2. Primers used in this study. Figure $\mathbf{S} 1$. The effect of new combination of CrtZ-CrtW on carotenoids profile. (a) Sketch map of CrtW-CrtZ expression cassette plasmids. CrtW-CrtZ expression cassette was carried by a multiple copy plasmid pRS425K. Expression modules for CrtW (TDH3p-crtW-TDH2t) and CrtZ (FBA1 p-crtZ-ADH1t) were arranged back-to-back with opposite transcriptional direction. Promoters, encoding sequences and terminators were presented as green, yellow and red, respectively. (b) The HPLC profile of strain SyBE_Sc118060 (blue) with BDC263_CrtW-Asp_CrtZ and SyBE_Sc307001 (red) with BDC263_CrtW-Aa_CrtZ. The black line indicated the profiles for mix-standard. Astaxanthin, zeaxanthin, canthaxanthin, lycopene and $\beta$-carotene were labeled as I, II, III, IV and V. Figure S2. The effect of CrtZ source on zeaxanthin yield. (a) Sketch map of CrtZ expression cassette plasmids. Promoters, encoding sequences and terminators were presented as green, yellow and red, respectively. (b) Zeaxanthin yield of strain SyBE_Sc307121 and SyBE_Sc307122. These two strains were generated from the same $\beta$-carotene producer SyBE_Sc118030 by individually expression of Aa_CrtZ or Asp_CrtZ. Figure S3. Effect of various plasma treatment times on the survival rate of SyBE_Sc307001. Data were pooled from three independent experiments. Figure S4. Cell growth and astaxanthin yield of ARTP mutants. Growth curve (a) and carotenoids profile (b) of strain SyBE_Sc307001 and its ARTP mutagenesis strains (SyBE_Sc2110M2, M4-M7) which did not achieve higher total carotenoids accumulation in YPD medium than the parent strain. A photograph was attached bellow the bar chart to illustrate visual color of the related strains. The error bars represent standard deviations calculated from duplicate experiments. The error bars represent standard deviations calculated from duplicate experiments. "Astaxanthin yield" was determined as "the astaxanthin content in single cell" with unit mg/g DCW. Figure S5. The copy numbers of plasmid pRS425K-BDC263_CrtW-Aa_CrtZ in strain SyBE_Sc307001, SyBE_Sc3070M1 and SyBE_Sc3070M3. Figure S6. PCR verification of the desired gene-deleted strains. (a) Sketch map of the design of PCR verify primers. One pair of the verified primers (I-F/I-R) were located upstream of the left homologous arm and downstream of the right homologous arm of the targeted gene; while another pair of the verified primers (II-F/ II-R) were located inside of the targeted gene. Primers I-F/I-R are able to amplify a clear band in the DNA poles for gene knocked-out stains; while Primers II-F/II-R are able to amplify a clear band only in the DNA poles for the parent stain. (b) Electrophoretic map of PCR products. To be notably, the primers I-F/I-R could amplify the band from control, which should be 5456 bp (for FLO9), 4988 bp (for CSS1), 4659 bp (for DAN4), 6724 bp (for YBR012W-B) and $6154 \mathrm{bp}(Y L R 410 W-B)$. However, the current extension time was just enough to amplify the band from the gene deleted strains but it was too short (or it was hard) to obtain the band from control. Figure S7. Growth curve of strains SyBE_Sc307001, SyBE_Sc2110M3 and gene deleted strains in SC medium. These gene knocked-out strains were generated from strain SyBE_Sc307001 by individual deletion of gene FLO9, CSS1, YLR410W-B, YBRO12W-B and DAN4, respectively. Figure S8. Astaxanthin yield of stain SyBE_SC2110M3 in each generation cultivated in SD medium.

\section{Abbreviations}

CrtZ: $\beta$-carotene hydroxylase; CrtW: $\beta$-carotene ketolase; Aa: Agrobacterium aurantiacum; Asp: Alcaligenes sp. strain PC-1; BDC263: Brevundimonas vesicularis DC263; ARTP: atmospheric and room temperature plasma. 


\section{Authors' contributions}

$J$ J, WX and $Y Y$ conceived of the study and drafted the manuscript. YW participated in design and coordination of the study. JJ and XG carried out the molecular genetic studies as well as performed the ARTP experiments. JJ and WX carried out the fed-batch fermentation experiments. MD assisted with heterologous module engineering. JJ, YW and BL conducted the comparative genome analysis. HL participated in HPLC analysis. YW and MD helped to draft the manuscript. WX supervised the whole research and revised the manuscript. All authors read and approved the final manuscript.

\section{Author details}

${ }^{1}$ Key Laboratory of Systems Bioengineering (Ministry of Education), Schoo of Chemical \& Engineering, Tianjin University, No. 92, Weijin Road, Nankai District, Tianjin 300072, People's Republic of China. ${ }^{2}$ SynBio Research Platform, Collaborative Innovation Center of Chemical Science and Engineering (Tianjin), Tianjin University, Tianjin 300072, People's Republic of China.

\section{Acknowledgements}

The authors are grateful for the financial support from the National Natural Science Foundation of China (21621004, 21676192 and 21676190), the Ministry of Science and Technology of China ("973" Program: 2014CB745100) and Innovative Talents and Platform Program of Tianjin (16PTSYJC00050 and 16PTGCCX00140).

\section{Competing interests}

The authors declare that they have no competing interests.

\section{Availability of supporting data}

Data will be made available from the corresponding author on reasonable request.

\section{Consent for publication}

All authors read and approved the final manuscript.

\section{Ethical approval and consent to participate} Not applicable.

\section{Funding}

The National Natural Science Foundation of China (21621004, 21676192 and 21676190), the Ministry of Science and Technology of China ("973" Program: 2014CB745100) and Innovative Talents and Platform Program of Tianjin (16PTSYJC00050 and 16PTGCCX00140).

\section{Publisher's Note}

Springer Nature remains neutral with regard to jurisdictional claims in published maps and institutional affiliations.

Received: 15 February 2018 Accepted: 14 August 2018 Published online: 23 August 2018

\section{References}

1. Ambati RR, Phang SM, Ravi S, Aswathanarayana RG. Astaxanthin: sources, extraction, stability, biological activities and its commercial applications-a review. Mar Drugs. 2014;12:128-52.

2. Igielska-Kalwat J, Goscianska J, Nowak I. Carotenoids as natural antioxidants. Postepy Hig Med Dosw (Online). 2015;69:418-28.

3. Kang CD, Lee JS, Park TH, Sim SJ. Comparison of heterotrophic and photoautotrophic induction on astaxanthin production by Haematococcus pluvialis. Appl Microbiol Biotechnol. 2005:68:237-41.

4. Ye VM, Bhatia SK. Pathway engineering strategies for production of beneficial carotenoids in microbial hosts. Biotechnol Lett. 2012;34:1405-14.

5. Lin YJ, Chang JJ, Lin HY, Thia C, Kao YY, Huang CC, Li WH. Metabolic engineering a yeast to produce astaxanthin. Bioresour Technol. 2017:245:899-905.

6. Scaife MA, Burja AM, Wright PC. Characterization of cyanobacterial beta-carotene ketolase and hydroxylase genes in Escherichia coli, and their application for astaxanthin biosynthesis. Biotechnol Bioeng. 2009;103:944-55.
7. Lemuth K, Steuer K, Albermann C. Engineering of a plasmid-free Escherichia coli strain for improved in vivo biosynthesis of astaxanthin. Microb Cell Fact. 2011:10:29.

8. Zelcbuch L, Antonovsky N, Bar-Even A, Levin-Karp A, Barenholz U, Dayagi M, Liebermeister W, Flamholz A, Noor E, Amram S, et al. Spanning highdimensional expression space using ribosome-binding site combinatorics. Nucleic Acids Res. 2013;41:e98.

9. Lu Q, Bu YF, Liu JZ. Metabolic engineering of Escherichia coli for producing astaxanthin as the predominant carotenoid. Mar Drugs. 2017;15:296.

10. Ma T, Zhou Y, Li X, Zhu F, Cheng Y, Liu Y, Deng Z, Liu T. Genome mining of astaxanthin biosynthetic genes from Sphingomonas sp. ATCC 55669 for heterologous overproduction in Escherichia coli. Biotechnol J. 2016;11:228-37.

11. Zhou P, Ye L, Xie W, Lv X, Yu H. Highly efficient biosynthesis of astaxanthin in Saccharomyces cerevisiae by integration and tuning of algal crtZ and bkt. Appl Microbiol Biotechnol. 2015;99:8419-28.

12. Zhou P, Xie W, Li A, Wang F, Yao Z, Bian Q, Zhu Y, Yu H, Ye L. Alleviation of metabolic bottleneck by combinatorial engineering enhanced astaxanthin synthesis in Saccharomyces cerevisiae. Enzyme Microb Technol. 2017:100:28-36.

13. Henke NA, Heider SA, Peters-Wendisch P, Wendisch VF. Production of the marine carotenoid astaxanthin by metabolically engineered Corynebacterium glutamicum. Mar Drugs. 2016;14:124.

14. Zhang C, Seow VY, Chen X, Too HP. Multidimensional heuristic process for high-yield production of astaxanthin and fragrance molecules in Escherichia coli. Nat Commun. 2018;9:1858.

15. Kim JH, Park HJ, Kim YH, Joo H, Lee SH, Lee JH. UV-induced mutagenesis of Nannochloropsis oculata for the increase of lipid accumulation and its characterization. Appl Chem Eng. 2013;24:155-60.

16. Cheng G, Xu J, Xia X, Guo Y, Xu K, Su C, Zhang W. Breeding L-arginine-producing strains by a novel mutagenesis method: atmospheric and room temperature plasma (ARTP). Prep Biochem Biotechnol. 2015;46:509-16.

17. Sandesh Kamath B, Vidhyavathi R, Sarada R, Ravishankar GA. Enhancement of carotenoids by mutation and stress induced carotenogenic genes in Haematococcus pluvialis mutants. Bioresour Technol. 2008;99:8667-73.

18. Zhang $X$, Zhang XF, Li HP, Wang LY, Zhang C, Xing XH, Bao CY. Atmospheric and room temperature plasma (ARTP) as a new powerful mutagenesis tool. Appl Microbiol Biotechnol. 2014;98:5387-96.

19. Oiang W, Ling-ran F, Luo W, Han-guang L, Lin W, Ya Z, Xiao-bin Y. Mutation breeding of lycopene-producing strain Blakeslea trispora by a novel atmospheric and room temperature plasma (ARTP). Appl Biochem Biotechnol. 2014;174:452-60.

20. Zhang C, Shen H, Zhang X, Yu X, Wang H, Xiao S, Wang J, Zhao ZK. Combined mutagenesis of Rhodosporidium toruloides for improved production of carotenoids and lipids. Biotechnol Lett. 2016;38:1733-8.

21. Martin JF, Gudina E, Barredo JL. Conversion of beta-carotene into astaxanthin: two separate enzymes or a bifunctional hydroxylase-ketolase protein? Microb Cell Fact. 2008;7:3.

22. Misawa N. Carotenoid beta-ring hydroxylase and ketolase from marine bacteria-promiscuous enzymes for synthesizing functional xanthophylls. Mar Drugs. 2011;9:757-71

23. Chang JJ, Thia C, Lin HY, Liu HL, Ho FJ, Wu JT, Shih MC, Li WH, Huang CC. Integrating an algal beta-carotene hydroxylase gene into a designed carotenoid-biosynthesis pathway increases carotenoid production in yeast. Bioresour Technol. 2015;184:2-8.

24. Bhataya A, Schmidt-Dannert C, Lee PC. Metabolic engineering of Pichia pastoris X-33 for lycopene production. Process Biochem. 2009:44:1095-102.

25. Wang R, Gu X, Yao M, Pan C, Liu H, Xiao W, Wang Y, Yuan Y. Engineering of $\beta$-carotene hydroxylase and ketolase for astaxanthin overproduction in Saccharomyces cerevisiae. Front Chem Sci Eng. 2017;11:89-99.

26. Fraser PD, Miura Y, Misawa N. In vitro characterization of astaxanthin biosynthetic enzymes. J Biol Chem. 1997;272:6128-35.

27. Chen Y, Xiao W, Wang Y, Liu H, Li X, Yuan Y. Lycopene overproduction in Saccharomyces cerevisiae through combining pathway engineering with host engineering. Microb Cell Fact. 2016;15:113.

28. Cherry JM, Hong EL, Amundsen C, Balakrishnan R, Binkley G, Chan ET, Christie KR, Costanzo MC, Dwight SS, Engel SR, et al. Saccharomyces genome database: the genomics resource of budding yeast. Nucleic Acids Res. 2012:40:D700-5. 
29. Mieczkowski PA, Lemoine FJ, Petes TD. Recombination between retrotransposons as a source of chromosome rearrangements in the yeast Saccharomyces cerevisiae. DNA Repair. 2006;5:1010-20.

30. Matmati N, Hannun YA. Thematic review series: sphingolipids. ISC1 (inositol phosphosphingolipid-phospholipase C), the yeast homologue of neutral sphingomyelinases. J Lipid Res. 2008;49:922-8.

31. Liu J, Zhu Y, Du G, Zhou J, Chen J. Exogenous ergosterol protects Saccharomyces cerevisiae from D-limonene stress. J Appl Microbiol. 2013:114:482-91.

32. Ahrazem O, Rubio-Moraga A, Berman J, Capell T, Christou P, Zhu C, Gomez-Gomez L. The carotenoid cleavage dioxygenase CCD2 catalysing the synthesis of crocetin in spring crocuses and saffron is a plastidial enzyme. New Phytol. 2016;209:650-63.

33. Rowlett VW, Mallampalli V, Karlstaedt A, Dowhan W, Taegtmeyer H, Margolin W, Vitrac H. Impact of membrane phospholipid alterations in Escherichia coli on cellular function and bacterial stress adaptation. J Bacteriol. 2017. https://doi.org/10.1128/JB.00849-16.

34. Tan Z, Khakbaz P, Chen Y, Lombardo J, Yoon JM, Shanks JV, Klauda JB, Jarboe LR. Engineering Escherichia coli membrane phospholipid head distribution improves tolerance and production of biorenewables. Metab Eng. 2017:44:1-12.

35. Liu YS, Wu JY. Hydrogen peroxide-induced astaxanthin biosynthesis and catalase activity in Xanthophyllomyces dendrorhous. Appl Microbiol Biotechnol. 2006;73:663-8.

36. Yang K, Duley ML, Zhu J. Metabolomics study reveals enhanced inhibition and metabolic dysregulation in Escherichia coli induced by Lactobacillus acidophilus-fermented black tea extract. J Agric Food Chem. 2018;66:1386-93.

37. Guo DS, Ji XJ, Ren LJ, Li GL, Yin FW, Huang H. Development of a real-time bioprocess monitoring method for docosahexaenoic acid production by Schizochytrium sp. Bioresour Technol. 2016;216:422-7.
38. Gietz RD. Yeast transformation by the LiAc/SS carrier DNA/PEG method. Methods Mol Biol. 2014;1205:1-12.

39. Hua X, Wang J, Wu Z, Zhang H, Li H, Xing X, Liu Z. A salt tolerant Enterobacter cloacae mutant for bioaugmentation of petroleum- and saltcontaminated soil. Biochem Eng J. 2010;49:201-6.

40. Liu J. Clostridium beijerinckii mutant obtained atmospheric pressure glow discharge generates enhanced electricity in a microbial fuel cell. Biotechnol Lett. 2015;37:95-100.

41. Jia B, Wu Y, Bing-Zhi Li, et al. Precise control of SCRaMbLE in synthetic haploid and diploid yeast. Nat Commun. 2018;9:1933.

42. Schmittgen TD, Livak KJ. Analyzing real-time PCR data by the comparative CT method. Nat Protoc. 2008;3:1101.

43. Zhi HS, Shuang XR, Xin QL, Jian X, Han Y, Guo PZ, Jin W. A preliminary study of the mechanism of nitrate-stimulated remarkable increase of rifamycin production in Amycolatopsis mediterranei U32 by RNA-seq. Microb Cell Fact. 2015;14:75.

44. Li H, Durbin R. Fast and accurate short read alignment with BurrowsWheeler transform. Bioinformatics. 2009:25:1754-60.

45. Li H, Handsaker B, Wysoker A, Fennell T, Ruan J, Homer N, Marth G, Abecasis $G$, Durbin R. Genome project data processing S: the sequence alignment/map format and SAMtools. Bioinformatics. 2009;25:2078-9.

46. Mortazavi A, Williams BA, McCue K, Schaeffer L, Wold B. Mapping and quantifying mammalian transcriptomes by RNA-Seq. Nat Methods. 2008;5:621-8.

47. Love MI, Huber W, Anders S. Moderated estimation of fold change and dispersion for RNA-seq data with DESeq2. Genome Biol. 2014;15:550.

48. Brachmann CB, Davies A, Cost GJ, Caputo E, Li J, Hieter P, Boeke JD. Designer deletion strains derived from Saccharomyces cerevisiae S288C: a useful set of strains and plasmids for PCR-mediated gene disruption and other applications. Yeast. 1998;14:115-32.
Ready to submit your research? Choose BMC and benefit from:

- fast, convenient online submission

- thorough peer review by experienced researchers in your field

- rapid publication on acceptance

- support for research data, including large and complex data types

- gold Open Access which fosters wider collaboration and increased citations

- maximum visibility for your research: over $100 \mathrm{M}$ website views per year

At $\mathrm{BMC}$, research is always in progress.

Learn more biomedcentral.com/submissions 